\title{
Comparing properties of the two microquasar GX 339-4 and 1E 1740.7-2942
}

\author{
Melania Del Santo* \\ INAF/IASF-Roma, Italy \\ E-mail: melania.delsantodiasf-roma.inaf.it \\ Julien Malzac, Elisabeth Jourdain
}

CNRS/CESR Toulouse, France

Angela Bazzano

INAF/IASF-Roma, Italy

\section{Laurent Bouchet, Jean-Pierre Roques}

CNRS/CESR Toulouse, France

\section{Tomaso Belloni}

INAF/OAB Merate, Italy

\section{Giovanni De Cesare, Pietro Ubertini, Memmo Federici}

INAF/IASF-Roma, Italy

\begin{abstract}
We report on INTEGRAL observations of the two microquasars GX 339-4 and 1E 1740.7-2942. Even though both sources are black hole candidates in LMXB systems, GX 339-4 has a transient behaviour, while the prototype of microquasars, 1E 1740.7-2942, is a persistent, even though variable source. We analysed a uniform data-set of GX 339-4 observations collected during the 2004 outburst using all INTEGRAL instruments. Observations were long enough to cover transitions between spectral states. Moreover, IBIS and SPI spectra of GX 339-4 showed a high energy excess with respect to pure thermal Comptonisation model, even during the hard/intermediate state. We analysed three years of SPI and IBIS data of 1E 1740.7-2942 during which two main states have been observed: the canonical low/hard state of black-hole candidates and a "dim" state, characterised by a $\sim 20$ times fainter emission. The low/hard state spectrum up to $\sim 600$ $\mathrm{keV}$ is well represented by a thermal Comptonisation plus an additional component modeling data above $200 \mathrm{keV}$. finally, we summarise results and make a comparison between the two sources.
\end{abstract}

7th INTEGRAL Workshop

September 8-11 2008

Copenhagen, Denmark

*Poster Presenter 

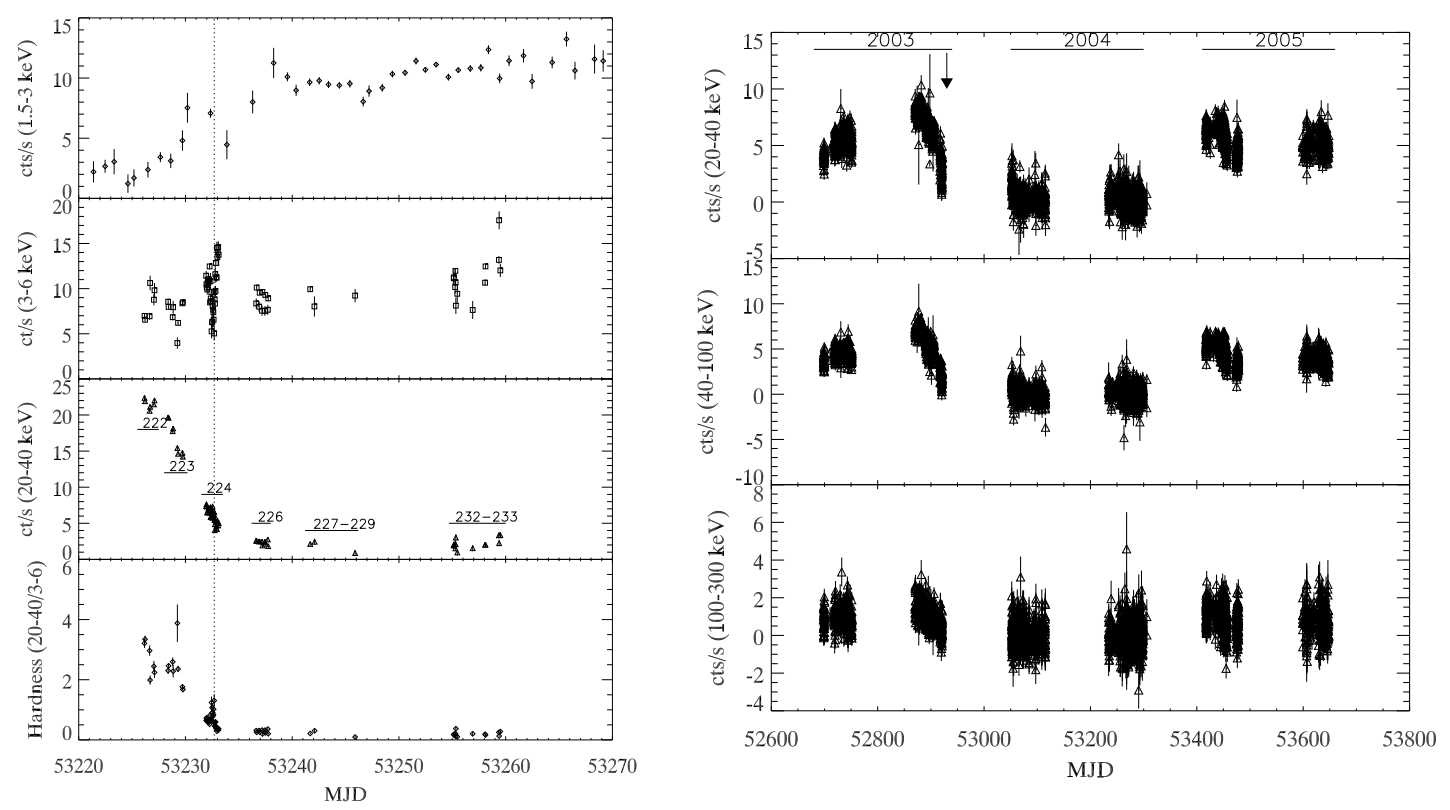

Figure 1: Left: GX 339-4 count rate in the energy ranges $1.5-3 \mathrm{keV}, 3-6 \mathrm{keV}, 20-40 \mathrm{keV}$ with ASM, JEM-X and IBIS/ISGRI, respectively. The related INTEGRAL orbits are also marked on the IBIS light curve. IBIS/ISGRI to JEM-X hardness ratio is shown in the bottom panel. Right: 1E 1740.7-2942 temporal behaviour observed with IBIS/ISGRI in 2003, 2004, 2005. In 2004 the source was very weak, both in hard $\mathrm{X}$-rays (2.6 mCrab in 20-40 keV) and in X-rays. In 2003 and 2005 it was most of the time in LHS, except in fall 2003, when it showed a soft/intermediate state before it quenched (arrow).

\section{Introduction}

Black hole candidates (BHCs) are known to show different spectral states in the $\mathrm{X}$ and $\gamma$-ray domain. The two main spectral states are the Low/Hard State (LHS), with a dominant Comptonised spectrum empirically described by a cut-off power-law (typically $\Gamma \sim 1.5$ and $E_{c u t} \sim 100 \mathrm{keV}$ ), and the High Soft State (HSS) with a spectrum described by a thermal component peaking at few keV and a high energy power-law much softer $(\Gamma>2.2)$ [19]. Intermediate spectral states with different contributions from the two components are also observed [20].

\section{INTEGRAL observations of the two microquasar}

\subsection{GX 339-4}

Before the launch of RXTE, the transient black-hole candidate (BHC) GX 339-4 was observed being very bright mostly in LHS for long time, until 1999 when it went into quiescence. Thereafter new outbursts occurred in 2002/2003 [17], in 2004 after one year in quiescence [5] and end 2006 [12]. The Hardness Intensity Diagrams (HID) of the three outbursts showed a similar pattern (i. e., the $q$-track of Homan \& Belloni 2005); a number of spectral states with different spectral and timing characteristics were observed in each a single outburst $[2,21,3,8,9]$.

The 2004 outburst was observed two times by the INTEGRAL satellite: during the first part of the hard X-ray activity (19 February-20 March) and during the decay of the hard X-ray flux, i. e. 9 

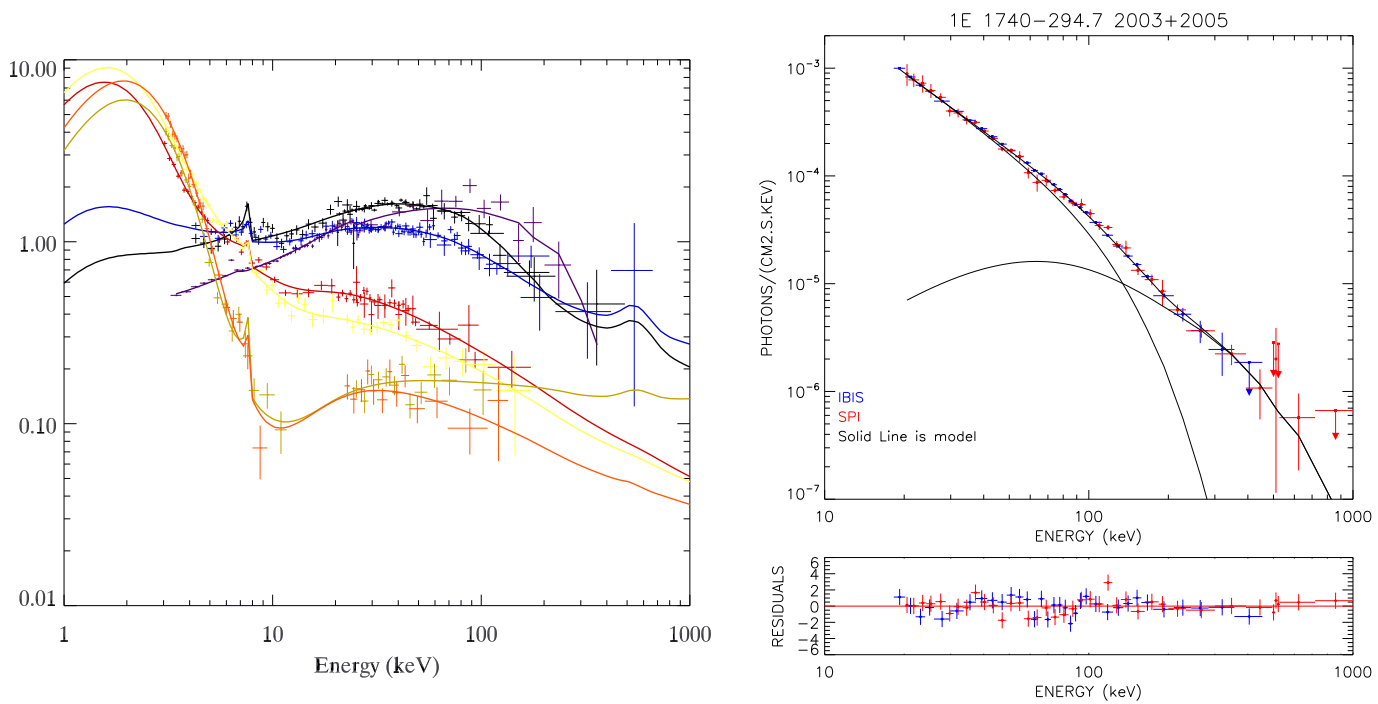

Figure 2: Left: Joint JEM-X, IBIS and SPI unfolded energy spectra (Model: EQPAIR) of GX 339-4 averaged during different spectral states in 2004: HIMS for spectra 1 (black), 2 (blue), 3 (red); SIMS for 4 (yellow); HSS for spectra 5 (green) and 7 (orange). In order to give a comparison with a pure Low/Hard state spectrum, we show a spectrum (violet) reported in Joinet et al. (2007). Right: 1E 1740.7-2942 spectrum observed by IBIS and SPI for $2003+2005$ observations. Solid line is a two comptonisation model ( $2 *$ comptt), with hotter temperature fixed to $100 \mathrm{keV}$. The other parameters are described in the text.

August-11 September. We used INTEGRAL data collected during the latter observations (see Fig. 1, left) in order to study the broad-band spectral evolution of GX 339-4 during a hard-to-soft state transition [8].

\subsection{E 1740.7-2942}

1E 1740.7-2942 spends most of the time in the canonical LH state of BHCs. In a few occasions soft spectral states have been observed [17] and in particular, in 2003 (see Fig. 1) INTEGRAL and RXTE observed an Intermediate/Soft spectral state occurred just before the source quenching [7].

Since its launch on October 17th 2002 INTEGRAL has been observing the Galactic Centre region, including the BHC 1E 1740.7-2942, two times per year, in the Spring and Fall visibility windows. We have analysed all public data collected between Spring 2003 and Fall 2005 by the spectrometer SPI and the imager IBIS. The useful data set consists in about 3500 exposures for a total useful time of 8 Ms along 6 periods (Spring and Fall, 2003, 2004 and 2005; see Fig.1, right).

Two main states have been observed: a canonical LHS at a flux level of $\sim 50 \mathrm{mCrab}$ and 60 mCrab in the 20-40 keV and 40-100 keV bands, respectively (Fig. 1, right), and a very-faint state (as in 2004) when the source was well below the ISGRI detection limit on the revolution time scale ( $\sim 200 \mathrm{ks})$ and detected at a level of 2.6-2.7 mCrab (14 $\sigma$ over $\sim 3$ months integration time). 


\section{Spectral analysis results}

\subsection{GX 339-4}

In the period 2004 August 9th-September 11th we observed GX 339-4 showing different spectral states, namely hard/intermediate (HIMS), soft/intermediate (SIMS), and HSS. According with the spectral analysis and timing, we divided data in seven groups obtaining seven averaged spectra. We refer to [8] for the scientific spectral parameters obtained by fitting joint JEM-X, IBIS and SPI spectra with EQPAIR [6] (Fig. 2, left). In the EQPAIR model, emission of the disc/corona system is modeled by a spherical hot plasma cloud with continuous acceleration of electrons illuminated by soft photons emitted by the accretion disc. At high energies the distribution of electrons is non-thermal, while at low energies a Maxwellian distribution with temperature $k T_{e}$ is established. Our detailed spectral analysis of time averaged spectra at different stages of the transition confirms that the spectral transition is driven by changes in the soft cooling photon flux in the corona associated with an increase of disc temperatures (leading to dramatic increase of disc luminosity). The measured disc temperature versus luminosity relation suggests that the internal disc radius decreases. In contrasts, the heating rate of the electrons in the corona appears to remain nearly constant [8].

Here we outline that fitting spectra of the HIMS, non-negligible values of $1_{\text {nth }} / l_{h}$ (compactness ratio between the electron acceleration and total power supplied to the plasma) resulted, indicating a non-thermal emission being requested by the data.

Using a simple thermal Comptonisation model, some residuals are present at high energy (Fig. 3), especially in the spectrum collected during the revolution 223 (blue spectrum in Fig. 2, left). In order to model the presence of a non-thermal component, we added a power-law with $\Gamma \sim 2$ to the pure thermal Comptonisation model (Fig. 4). This led to an improvement of the $\chi^{2}$ that is highly significant with the F-test probability as $p_{\text {compps }}=6.6 \times 10^{-2}$ (see Del Santo et al. 2008 for a complete discussion).

\subsection{E 1740.7-2942}

In spite of the flux variability, in 2003 and 2005 1E 1740.7-2942 was mostly in Low/Hard state with the same spectral parameters [7, 4].

Due to the modest SPI angular resolution $\left(\sim 2.6^{\circ}\right)$, the spectrum directly extracted at 1E 1740.7-2942 position may contain contributions from other weak/close/"not detected" sources. Nevertheless, it is possible to obtain the emission spectrum of 1E 1740.7-2942 from SPI data with the information provided by IBIS/ISGRI (angular resolution $\sim 12^{\prime}$ ). After the SPI data correction by removing emission of the nearby sources (see [4] for a complete data analysis description), we have built averaged spectra for 2003 and 2005, separately.

In a second step, we averaged a LHS spectrum collected during the whole analysed period. It allowed us to achieve a better statistics at high-energy and eventually give better constraints to the spectral parameters. Spectral fitting was performed with a thermal Comptonisation model (COMPTT, Titarchuk 1994), being the mechanism expected to play the major role in our energy domain (Tab. 1). We obtain electron temperatures of $50 \mathrm{keV}$ with optical depths close to 1 that are quite canonical values for this class of objects. However, the total $(2003+2005)$ averaged spectrum 


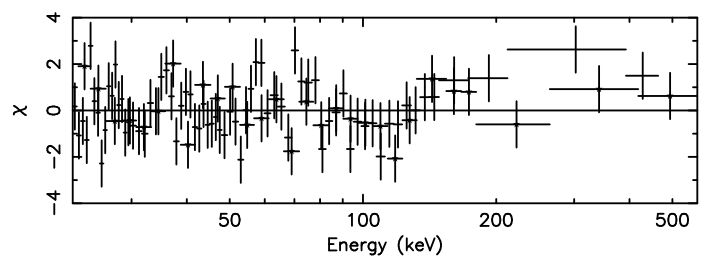

Figure 3: Residuals obtained with a simple thermal Comptonisation model (COMPPS) by fitting IBIS and SPI spectra of GX 339-4 during orbit 223.
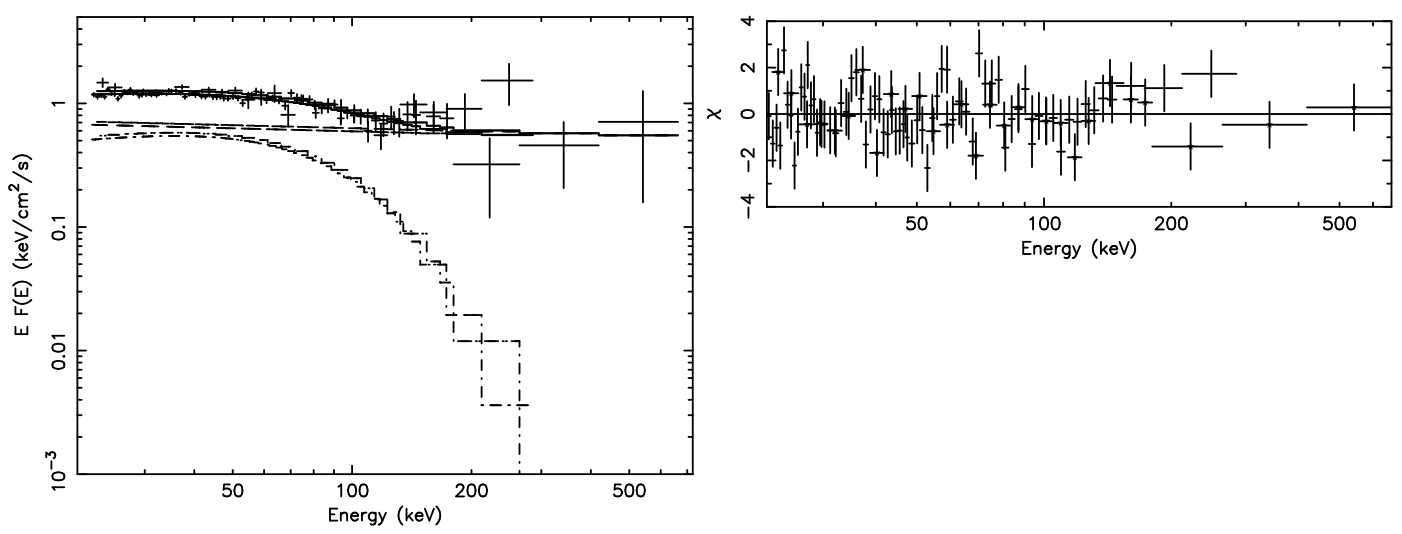

Figure 4: GX 339-4 energy spectrum and residuals of rev 223 fitted with a Comptonisation model (COMPPS) plus power-law.

gives high $\chi_{v}^{2}$ value (1.8 for 69 dof), which, together with an excess of emission relatively to the proposed model, suggests the presence of a supplementary component to explain data points above $200 \mathrm{keV}$.

Adding a power-law component $(\Gamma=1.9 \pm 0.1)$ when fitting the high energy data, it resulted a $\chi_{v}^{2}$ close to 1 . The plasma temperature is thus decreased as $27 \pm 2.2 \mathrm{keV}$ with a value of $\tau$ of 1.9 \pm 0.25 . Even though this two components model is quite reasonable, with a classical explanation of a non-thermal process responsible of the power-law emission, we tested an alternative scenario with only thermal mechanisms involved (Tab. 1). Indeed, a two (thermal) Comptonisation model gives a similarly acceptable (in term of $\chi^{2}$ ) description of the spectrum. However parameters are very poorly constrain, so that we had to freeze temperature of the second electrons population at $100 \mathrm{keV}$. Moreover, we consider that this population comptonises photons coming from the first Comptonising region, i. e. $\left(\mathrm{kT}_{\text {seed }}\right)_{2}=\left(\mathrm{kT}_{e}\right)_{1} \sim 30 \mathrm{keV}$. The optical depths of both regions are compatible (Tab. 1) while the $\chi_{v}^{2}=1.07$ (67 dof) leads to an F-test probability of $\sim 10^{-8}$ for the existence of such a second component. Results corresponding to this scenario are displayed in Fig. 2 (Right).

\section{Discussion}

GX 339-4: we observed spectral transitions as driven by changes in the soft cooling photon flux in the corona associated with an increase of disc temperatures. This gives rise to a dramatic increase of the disc luminosity. The measured disc temperature versus luminosity relation suggests 
Table 1: The best fit parameters for the combined ISGRI and SPI 1E 1740.7-2942 spectra with Comptonisation model (COMPTT, Titarchuk 1994). T0 is fixed to $0.3 \mathrm{keV}$. For the $2003+2005$ spectrum, the second line corresponds to a 2 Comptonisation model $\left(\left(\mathrm{kT}_{e}\right)_{1}, \tau_{1},\left(\mathrm{kT}_{e}\right)_{2}, \tau_{2}\right)$ with the 2 nd seed photon temperature fixed to $\left(\mathrm{kT}_{e}\right)_{1}$ and $\left(\mathrm{kT}_{e}\right)_{2}$ fixed to $100 \mathrm{keV}$.

\begin{tabular}{lcccccc}
\hline \hline period & $\begin{array}{c}\left(\mathrm{kT}_{e}\right)_{1} \\
\mathrm{keV}\end{array}$ & $\tau_{1}$ & $\begin{array}{c}\left(\mathrm{kT}_{e}\right)_{2} \\
\mathrm{keV}\end{array}$ & $\tau_{2}$ & $\chi_{v}^{2}(\mathrm{dof})$ & F-test $^{(1)}$ \\
& $50.1 \pm 2.6$ & $1.00 \pm 0.06$ & & & $1.1(69)$ & \\
2003 & $45.4 \pm 2.7$ & $1.10 \pm 0.08$ & & & $1.35(69)$ & \\
$2003+2005$ & $48.1 \pm 2.0$ & $1.06 \pm 0.05$ & & & $1.8(69)$ & \\
$2003+2005$ & $29.4 \pm 3.1$ & $1.6 \pm 0.1$ & 100 & $2.2 \pm 0.8$ & $1.07(67)$ & $10^{-8}$ \\
\hline
\end{tabular}

${ }^{(1)}$ for the presence of a second Comptonisation component.

that the internal disc radius decreases. In contrasts, the heating rate of the electrons in the corona appears to remain nearly constant.

Although other models such as dynamic accretion disc corona model cannot be ruled out, our results are consistent with the so-called truncated disc model [10]. Moreover, in all GX 339-4 spectra, including those in (HIMS), we found a significant contribution from a non-thermal component. This component appears as a high energy excess above the pure thermal Comptonisation spectrum. We associated this component with the presence of a non-thermal tail in the distribution of the Comptonising electron.

1E 1740.7-2942: we have observed two main states, namely the canonical low/hard state of black-hole candidates and a "dim" state, characterised by a $\sim 20$ times fainter emission, detected only below $50 \mathrm{keV}$ and only when adding more than $1 \mathrm{Ms}$ of data. For the first time the continuum of the low/hard state has been measured up to $\sim 600 \mathrm{keV}$ with a spectrum that is well represented by a thermal Comptonisation plus an additional component necessary to fit the data above 200 $\mathrm{keV}$ [4]. This high energy component could be related to non-thermal processes, even though this interpretation is more consistent with intermediate and/or soft states [21]. Alternatively, we show that a model composed by two thermal Comptonisations provides an equally representative description of the data: the temperature of the first population of electrons results as $\sim 30 \mathrm{keV}$ while the second, $\mathrm{kT}_{2}$ is fixed at $100 \mathrm{keV}$. This scenario could be the result of spatial/temporal variations in plasma parameters [14].

\section{Comparison:}

- GX 339-4 is a transient BHC which shows frequent outbursts at different peak luminosity levels [3, 8, 9], while 1E $1740.7-2942$ is a persistent source which periodically undergoes a dim state.

- GX 339-4 shows a complete set of spectral states, as well as fast state transitions (LHSHIMS-SIMS-HSS) at difference flux levels, while 1E 1740.7-2942 is observed to be most of the time in the canonical LHS at different luminosities and only before the quenching it shows a temporary intermediate/soft state [7, 17];

- we have observed high energy tails in both sources that may be interpreted as non-thermal 
components; alternatively, a model with two thermal Comptonisation components can be invoked for the Low/Hard state of 1E 1740.7-2942.

\section{Acknowledgments}

MDS is supported by the Italian Space Agency (ASI), via contract ASI/INTEGRAL I/008/07/0. As one of the thousands Italian researchers with a long-term temporary position, MDS acknowledges the support of Nature $(455,835-836)$ and thanks the Editors for increasing the international awareness of the current critical situation of the Italian Research. JM acknowledges support from CNRS. TB acknowledges support from the International Space Science Institute (ISSI) and from ASI via contract I/088/06/0.

\section{References}

[1] Belloni T., et al., 2004, ATel 236

[2] Belloni T., et al., 2005, A\&A, 440, 207

[3] Belloni T., et al., 2006, MNRAS, 367, 1113

[4] Bouchet L., et al., 2008, ApJ, accepted

[5] Buxton M., Bailyn C., 2004, ATel 270

[6] Coppi P. S., 1999, ASP Conf. Ser. 161: High Energy Processes in Accreting Black Holes, 161, 375

[7] Del Santo M., et al., 2005, A\&A, 433, 613

[8] Del Santo M., et al., 2008, MNRAS, 390, 227

[9] Del Santo M., et al., 2009, MNRAS, 392, 992

[10] Done C., Gierliński M., Kubota A., 2007, A\&A Review, 15, 1

[11] Homan J., Belloni T., 2005, in T. J. Maccarone, R. P. Fender, L. C. Ho eds, "From X-ray Binaries to Quasars: Black Hole Accretion on All Mass Scales", Ap\&SS, 300, 107

[12] Krimm H. A., et al., 2006, ATel \#968

[13] Joinet A., et al., 2007, ApJ, 657, 400

[14] Malzac J., \& Jourdain E., 2000, A\&A, 359, 843

[15] Miller J. M., et al., 2007, ATel 980

[16] Miyamoto S., Kimura K., Kitamoto S., Dotani T., Ebisawa K., 1991, ApJ, 383, 784

[17] Smith D. M., et al., 2002, ATel \#95

[18] Titarchuk, L. 1994, ApJ, 434, 313

[19] Zdziarski A. A., 2000, Proceedings of IAU Symposium, 195, 153

[20] Zdziarski A. A., Gierkiński M., 2004, Progress of theoretical Physics, 155, 99

[21] Zdziarski A. A., et al., 2004, MNRAS, 351, 791 